\title{
The influence of bladder management on fertility in spinal cord injured males
}

\author{
SB Rutkowski ${ }^{1}$, JW Middleton ${ }^{2}$, G Truman ${ }^{4}$, DL Hagen ${ }^{3}$ and JP Ryan ${ }^{5}$ \\ ${ }^{1}$ Director, ${ }^{2}$ Staff Specialist, ${ }^{3}$ Clinical Nurse Specialist and Clinical Spinal Nurse, Spinal Injuries Unit, Royal \\ North Shore Hospital, St Leonards, New South Wales 2065; ${ }^{4}$ Research Officer, Rehabilitation Studies Unit, \\ Department of Medicine, The University of Sydney, PO Box 6, Ryde, New South Wales 2112; ${ }^{5}$ Human \\ Reproduction Unit, Royal North Shore Hospital, St Leonards, New South Wales 2065, Australia
}

\begin{abstract}
Male infertility is a well recognised problem following spinal cord injury. The techniques of vibration induced ejaculation and transrectal electroejaculation have significantly increased the likelihood of sperm retrieval in spinal cord injured males; however, the reproductive capacity remains markedly reduced due to poor semen quality. The Spinal Injuries Unit at Royal North Shore Hospital has developed a programme to achieve seminal emission and enhance fertility. This study analysed the results of the first sample obtained at stimulation in 70 spinal cord injured males with respect to procedure performed, neurological level, completeness of lesion, bladder management, infection, age and duration since injury. Our study demonstrated that bladder management and neurological level were significant factors affecting the presence of motile sperm. Individuals managing their neuropathic bladder by catheter (intermittent self-catheterisation, indwelling urethral or suprapubic catheter) had significantly enhanced semen quality compared to those voiding by reflex or straining. Differences were also noted within the catheter group itself with intermittent self-catheterisation achieving a higher percentage of motile sperm present.
\end{abstract}

Keywords: fertility; bladder management; vibroejaculation; electroejaculation; emission; neurological level

\section{Introduction}

Following spinal cord injury (SCI), only an estimated $5 \%$ of men retain a natural unaided reproductive capacity. Previous studies suggest that without intervention between 5 and $18 \%$ of men with a complete lesion, depending on whether an upper motor neuron or lower motor neuron lesion, are able to ejaculate. ${ }^{1-3}$ Irrespective of whether ejaculation is aided or unaided, semen quality has usually been poor, particularly with regard to sperm motility. Linsenmeyer and Perkash ${ }^{4}$ in an extensive review of the literature identified eight factors that may contribute to the poor semen quality in SCI men: recurrent urinary tract infections, ${ }^{5}$ type of bladder management, ${ }^{6}$ stasis of prostatic fluid, testicular hyperthermia, abnormal testicular histology, possible changes in the hypothalamic-pituitary-testicular axis, possible sperm antibodies and long term use of various medications. In concluding their paper Linsenmeyer and Perkash ${ }^{4}$ stated that one future goal is to '... identify which, if any, of the many causes of poor semen quality after SCI are most important, and then to develop strategies to prevent them from occurring' (p752).

Over the past 5 years the spinal unit at Royal North Shore Hospital has been developing a service to enhance fertility capacity for spinal cord injured individuals. We have used the technique of vibroejacula- tion since November 1987 and electroejaculation since April 1991 following research and development of suitable equipment. Initially, the prime objective of our 'fertility clinic' was to explore the possibility of retrieving semen in spinal cord injured men. More recently, we have focused our attention on fertility enhancement with the assistance of the human reproduction unit and their in vitro fertilization (IVF) techniques including intracytoplasmic sperm injection (ICSI).

In this clinical setting we have observed a relationship between bladder management and semen quality, which we have actively sought to modify in an attempt to enhance fertility potential. This study will examine our results.

\section{Method}

Subjects

Results of vibroejaculation and electroejaculation procedures in 70 men with spinal cord injury were examined retrospectively. The patients studied were all those who enrolled for assessment and, in some cases, enhancement of fertility as outpatients at Royal North Shore Hospital between November 1988 and January 1994. Most, but not all, had been managed acutely in the spinal injuries unit of Royal North Shore Hospital. 
Informed consent was obtained prior to either procedure being performed. The median interval from spinal cord injury to procedure was 6 years with a range of 3 months to 29 years and the median age was 30 years with a range of 19 to 59 years. Of the 70 patients enrolled, there were 36 with neurological levels between $\mathrm{C} 1-8,19$ between T1-9, and 15 between T10-L2.

\section{Equipment and techniques}

Vibroejaculation The procedure of vibroejaculation ${ }^{7}$ was performed to induce reflex ejaculation with either a Matoba ME 25 (Matoba, Japan) or Thrive 9000 (Daito Electric Machine Ind. Co. Ltd, Osaka, Japan) commercially available vibrator. The vibrator was applied to the lower surface of the glans penis between the meatus and the frenulum. The duration of the vibratory stimulus was typically $3 \frac{1}{2} \mathrm{~min}$ on with rests of $1 \mathrm{~min}$ and the cycle repeated three times if necessary. Ejaculation was usually induced within $30 \mathrm{~s}$ of commencement of a cycle of vibratory stimulus. This was suitable only for lesions above the spinal cord centre for emission (ie neurological level above T10). The blood pressure was closely monitored due to the frequent occurence of autonomic dysreflexia in those with neurological levels above T6, most of whom required prophylaxis using sublingual nifedipine. If the technique achieved a reflexly induced ejaculation an antegrade specimen of semen was obtained. A urine sample was collected (usually by catheterization) for microscopic examination of retrograde ejaculation only in those patients in whom no antegrade specimen was obtained.

Electroejaculation Electrostimulation was performed using the Eljac (Bosshard Biomed, Sydney, Australia) battery operated device with the patient placed in the lateral decubitus position. Prior to each procedure, the rectal cavity was examined to determine appropriate probe position and to exclude the presence of any faecal matter or abnormality. Those with a spinal cord level above T6 were managed for autonomic dysreflexia as for vibroejaculation and blood pressure was again closely monitored throughout. Originally, before commencing electrical stimulation, the patient's bladder was emptied by a $12 \mathrm{Fg}$ disposable catheter and flushed with a buffer solution prepared by the IVF laboratory at Royal North Shore Hospital. Ten ml buffer solution was then instilled into the bladder before the catheter was removed. More recently, it has become our practice to leave an all silicone ballooned catheter in situ and apply gentle traction to it to tamponade the bladder neck in an attempt to prevent retrograde flow of semen. Once the procedure has been completed and the rectal probe removed, digital prostatic massage followed by perineal and urethral milking ensures complete retrieval of the emission. A urine sample was routinely collected to examine for retrograde emission.

The electrical stimulation pattern most often used was sinusoidal with a frequency of either 10 or $50 \mathrm{~Hz}$; voltage varied between 22 and $30 \mathrm{~V}$ and a current up to $320 \mathrm{~m} \mathrm{~A}$. The ramping and peaking of the voltage was adjusted according to the individual patient's response (observing various external signs of response such as erection, hamstring and abdominal muscle spasm) and prior experience in the case of repeat stimulations. At each procedure there was a maximum of three cycles of stimulation with a maximum duration of $45 \mathrm{~s}$.

A protocol regarding choice of the initial procedure performed has been developed with experience on clinical grounds. Until April 1991, vibroejaculation was the only procedure available. However, since then, once the technique became reliable, electroejaculation has been used for the initial stimulation in patients with neurological levels above T6 where better control of autonomic dysreflexia is possible and in those where vibroejaculation was previously unsuccessful.

Statistical analysis The results of the first sample obtained using either vibroejaculation or electroejaculation were examined with respect to individual factors including procedure, neurological level, completeness of lesion, bladder management, infection, age and duration since injury. Procedure was either vibroejaculation or electroejaculation. Neurological level was divided into one of three groups, C1-8, T1-9 and T10 and below. Each lesion was also classified on the basis of either complete (Frankel A) or incomplete (Frankel B-D) neurological impairment. Bladder management was divided into one of three categories: (1) clean intermittent self-catheterisation (CISC), (2) an indwelling urethral catheter (IDC) or suprapubic catheter (SPC) or (3) drainage without a catheter (including voiding by reflex or straining). Infection was scored as being present or absent in the sample. Infection was defined as a predominant pure growth of one bacteria, ie not a mixed growth, with greater than $10^{6}$ organisms $\mathrm{ml}^{-1}$ and a white cell count greater than $100 \times 10^{6} \mathrm{ml}^{-1}$. For this study, the presence or absence of motile spermatozoa were chosen as outcome measures. Logistic regression was used to determine the significance of the effect of factors on outcome.

\section{Results}

Vibroejaculation was used in 36 patients and electroejaculation in 34 patients to obtain the first laboratory sample that was examined. Nine of the patients in the electroejaculation group had failed to achieve an ejaculation using vibroejaculation previously.

Neurological level, procedure, completeness of lesion, method of bladder management and infections present or absent in the sample were entered into the logistic regression as categorical variables, and age and years since injury were entered as continuous variables. Univariate analyses were used in the initial screening of the variables, and variables with a $P$ value of greater than 0.25 were not considered in the logistic regression. ${ }^{8}$ 
Presence or absence of motile spermatozoa Univariate analyses revealed that all variables except completeness of lesion were suitable for inclusion in the logistic regression (Table 1 ).

Four variables (neurological level, procedure, method of bladder management, and presence or absence of infection in the sample) were entered with age and years since injury into a maximum likelihood test of logistic regression ${ }^{9}$ with an inclusion criterion $P<0.05$. Two variables were found to be significant predictors: neurological level and method of bladder management. These variables correctly predicted $70.00 \%$ of the outcome of the patients' samples. The remaining variables had $P$ values that were well outside the inclusion criterion of $P<0.05$ (see Table 2 ).

As the neurological level studied became more caudal, the proportion of patients with motile spermatozoa present in their sample decreased. The use of a catheter for bladder management instead of a noncatheter method resulted in a significantly higher proportion of patients with motile spermatozoa in their sample. The group using CISC had the highest proportion of motile spermatozoa present. The effect of these two variables on the predicted presence or absence of motile sperm in a patient's sample is shown in Figure 1. There appears to be a linear relationship between neurological level and likelihood of the presence of motile spermatozoa, while the difference between the two catheter and non-catheter groups remains about the same across the three different neurological levels.

\section{Discussion}

This study has demonstrated that both method of bladder management and neurological level were significant factors affecting the presence or absence of
Bladder management and male fertility SB Rutkowski et al

Table 2 Predictors of the presence or absence of motile spermatozoa in patients' samples

\begin{tabular}{lccc}
\hline Variable & Wald & $d f$ & $P$ \\
\hline Significant predictors & & & \\
$\quad$ Neurolgical level & 9.8777 & 2 & 0.0072 \\
$\quad$ Bladder control method & 8.9374 & 2 & 0.0115 \\
Non-significant variables & & & \\
$\quad$ Years since injury & 0.0155 & 1 & 0.9010 \\
Age & 0.1179 & 1 & 0.7313 \\
Procedure & 0.2277 & 1 & 0.6332 \\
Infection & 3.0500 & 1 & 0.0807 \\
\hline
\end{tabular}

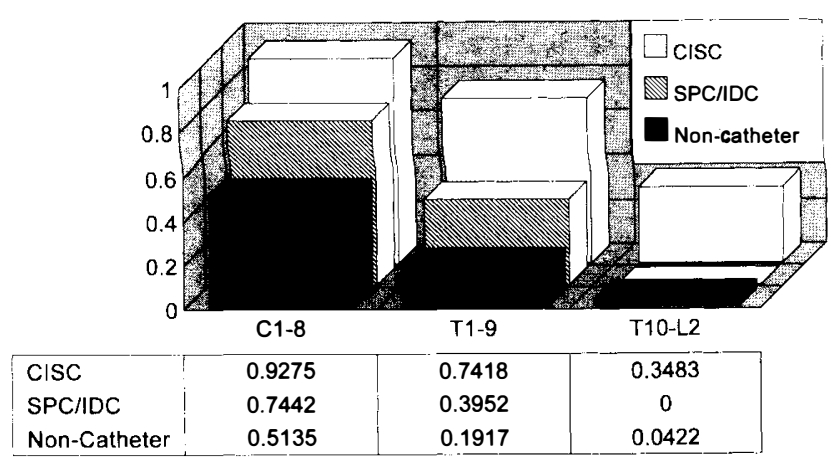

From First Sample

Figure 1 Predicted probability of motile sperm. The effect of neurological level and bladder management

motile sperm in semen samples collected from men after spinal cord injury. It is important to note that this study has only analysed the results of the sample obtained at the first stimulation using either vibroejaculation or electroejaculation. In fact nine of those who

Table 1 Univariate analyses of factors affecting the presence or absence of motile spermatozoa in the sample

\begin{tabular}{|c|c|c|c|c|}
\hline Variable & $\begin{array}{c}\text { Motile spermatozoa } \\
\text { present } \\
(n)\end{array}$ & $\begin{array}{c}\text { Motile spermatozoa } \\
\text { absent } \\
(n)\end{array}$ & $\begin{array}{c}X^{2} \\
(d f)\end{array}$ & $P$ \\
\hline \multicolumn{5}{|l|}{ Neurological level } \\
\hline $\mathrm{C} 1-8$ & 23 & 13 & \multirow{3}{*}{$\begin{array}{c}6.04 \\
(2)\end{array}$} & \multirow{3}{*}{0.05} \\
\hline T1-9 & 9 & 10 & & \\
\hline T10 \& below & 4 & 11 & & \\
\hline \multicolumn{5}{|l|}{ Procedure } \\
\hline Vibroejaculation & 22 & 14 & 2.04 & \multirow{2}{*}{0.15} \\
\hline Electrostimulation & 14 & 20 & (1) & \\
\hline \multicolumn{5}{|l|}{ Completeness of lesion } \\
\hline Complete & 25 & 23 & 0.00 & \multirow[t]{2}{*}{1.00} \\
\hline Incomplete & 11 & 11 & $(1)$ & \\
\hline \multicolumn{5}{|c|}{ Method of bladder mangement } \\
\hline CISC & 17 & 10 & 4.64 & \multirow[t]{3}{*}{0.10} \\
\hline SPC or IDC & 6 & 3 & $(2)$ & \\
\hline Non-catheter & 13 & 21 & & \\
\hline \multicolumn{5}{|l|}{ Infection in sample } \\
\hline Present & 6 & 11 & 1.56 & \multirow[t]{2}{*}{0.21} \\
\hline Absent & 30 & 23 & (1) & \\
\hline
\end{tabular}


underwent electroejaculation had previously failed to achieve an ejaculation with vibration prior to the commencement of this study. We acknowledge that results obtained at initial stimulation may not always correlate well with eventual success of the procedure or ultimate quality of the semen. Several previous authors ${ }^{6,10}$ have shown an improvement in semen quality with several successive stimulations which is also in accordance with our own observations.

We postulate that a high pressure neuropathic bladder results in reflux of urine into the genital tract and that active management to reverse this can result in a significant improvement in fertility outcome. Ohl et $a l^{6}$ have demonstrated such reflux of urine into the ejaculatory ducts, seminal vesicles and vas deferens under high pressure voiding to empty a neuropathic bladder on a videourodynamic study. Crich and Jequier ${ }^{11}$ have noted the deleterious effects of urine on sperm motility with at least a $50 \%$ reduction within 5 min of contact.

An essential part of any programme to enhance fertility potential in spinal cord injured men is fully to assess and optimize the method of bladder management to ensure low pressure emptying and avoidance of infections.

\section{Conclusion}

While it may not be possible to influence all the factors which may contribute to poor semen quality in spinal cord injured individuals, it is possible to alter the method of bladder management to ensure low pressure drainage of the neuropathic bladder and enhance fertility potential. We recommend some form of catheter drainage to achieve this, with CISC the method of choice where possible.

\section{Acknowledgement}

The authors wish to thank Ms Frances Page-de Mars for her kind assistance in preparing this manuscript.

\section{References}

1 Horne MW, Paull DP, Munro D. Fertility studies in the human male with traumatic injuries of the spinal cord and cauda equina. New Engl J Med 1948; 237: 959-961.

2 Talbot HS. The sexual function in paraplegia. J Urol 1955; 73: 91-100.

3 Bors E, Comarr E. Neurological disturbances of sexual function with special reference to 529 patients with spinal cord injury. Urol Surv 1960; 10: 191-222.

4 Linsenmeyer TA, Perkash I. Infertility in men with spinal cord injury. Arch Phys Med Rehabil 1991; 72: 747-754.

5 Wolff $\mathrm{H}$ et al. Leukocytospermia associated with poor semen quality. Fertil Steril 1990; 53: 528-536.

6 Ohl DA et al. Predictors of success in electroejaculation of spinal cord injured men. J Urol 142: 1483-1486.

7 Brindley GS $(1984)$ The fertility of men with spinal injuries. Paraplegia 1984; 22: 337-348.

8 Hosmer DW, Lemeshow S. Applied logistic regression. John Wiley \& Sons: New York, 1989.

9 Norusis MJ. SPSS for Windows Advanced Statistics Release 6.0. SPSS Inc: Chicago, 1993.

10 Brindley GS. Physiology of erection and management of paraplegic infertility. In: TB Hargreave (ed). Male Infertility. Springer Verlag: Berlin, 1983, pp 261-279

11 Crich JP, Jequier AM. Infertility in men with retrograde ejaculation: The action of urine on sperm motility, and a simple method for achieving antegrade ejaculation. Fertil Steril 1978; 30: 5: $572-576$. 